\title{
LEPTO and Polarized SIDIS
}

\author{
Aram Kotzinian \\ Dipartimento di Fisica Generale, Università di Torino \\ and INFN, Sezione di Torino, Via P. Giuria 1, I-10125 Torino, Italy \\ and Yerevan Physics Institute, 375036 Yerevan, Armenia \\ and JINR, 141980 Dubna, Russia \\ email: aram.kotzinian@cern.ch
}

March 7, 2019

\begin{abstract}
Hadron production in the LEPTO event generator is modeled as a product of distribution functions and LUND hadronization functions (LHF) weighted by the hard scattering cross sections. The description of polarized SIDIS within this formalism includes a new nonperturbative input - polarized LHF's. It is shown that this approach does not exactly correspond to the commonly adopted one with the independent fragmentation functions. The purity method used by the HERMES collaboration mixes up the two approaches and ignores the contributions from polarized LHF's. This method cannot be considered a precise tool for the extraction of polarized quark distributions from measured SIDIS asymmetries.
\end{abstract}

\section{Introduction}

Recently, the important issue of the extraction of polarized quark distributions was again addressed by the HERMES collaboration [1. They have used the LO analysis of semi inclusive deep inelastic scattering (SIDIS) based on the so called purity method.

It is evident that the theoretical description of SIDIS is much more complicated than that of DIS owing to our poor knowledge of the nonperturbative hadronization mechanism. Traditionally, one distinguishes two regions for hadron production: the current fragmentation region, $x_{F}>0$ and the target fragmentation region, $x_{F}<0^{1}$. The common assumption is that hadrons in the current fragmentation region with $z>0.2$ are produced in the independent quark fragmentation.

To perform flavor decomposition of polarized quark distributions, the purity method has been used in the HERMES analysis [1]. In the LO approximation of perturbative

\footnotetext{
${ }^{1}$ We use the standard SIDIS notations and variables like in [1].
} 
QCD the SIDIS cross section for unpolarized target is given as

$$
\sigma^{h}\left(x, Q^{2}, z\right) \propto\left(1+(1-y)^{2}\right) \sum_{q} e_{q}^{2} q\left(x, Q^{2}\right) D_{q}^{h}\left(z, Q^{2}\right)
$$

and for polarized beam and target

$$
\Delta \sigma^{h}\left(x, Q^{2}, z\right) \propto\left(1-(1-y)^{2}\right) \sum_{q} e_{q}^{2} \Delta q\left(x, Q^{2}\right) D_{q}^{h}\left(z, Q^{2}\right) .
$$

Then the virtual photon asymmetry for hadron $h$ production is given by

$$
A_{1}^{h}\left(x, Q^{2}, z\right)=\frac{\sum_{q} e_{q}^{2} \Delta q\left(x, Q^{2}\right) D_{q}^{h}\left(z, Q^{2}\right)}{\sum_{q} e_{q}^{2} q\left(x, Q^{2}\right) D_{q}^{h}\left(z, Q^{2}\right)} .
$$

This equation can be rewritten as follows:

$$
A_{1}^{h}\left(x, Q^{2}, z\right)=\sum_{q} \mathcal{P}_{q}^{h}\left(x, Q^{2}, z\right) \frac{\Delta q\left(x, Q^{2}\right)}{q\left(x, Q^{2}\right)}
$$

where the quark polarizations $(\Delta q / q)$ are factored out and the purities, $\mathcal{P}_{q}^{h}$, are defined as

$$
\mathcal{P}_{q}^{h}\left(x, Q^{2}, z\right)=\frac{e_{q}^{2} q\left(x, Q^{2}\right) D_{q}^{h}\left(z, Q^{2}\right)}{\sum_{q^{\prime}} e_{q^{\prime}}^{2} q^{\prime}\left(x, Q^{2}\right) D_{q^{\prime}}^{h}\left(z, Q^{2}\right)} .
$$

and calculated using the Monte Carlo unpolarized event generator LEPTO [2]. Then, using the asymmetries, measured for different hadrons one can find $\Delta q(x)$ by solving Eq. (44).

The main assumption of this method is that the hadronization mechanism in LEPTO is the same as in naïve picture of SIDIS where all hadrons in the current fragmentation region with $z>0.2$ are produced in independent quark fragmentation and there are no additional terms in both the numerator and the denominator of Eqs. (3).

This assumption is not proven at moderate energies. As it will be demonstrated in Sec. 2, the properties of the "quark fragmentation functions" extracted from generated LEPTO samples are in contradiction with generally accepted properties of independent quark fragmentation functions. The reason for this discrepancy is indicated. The generalization of the parton model expression for polarized SIDIS is given in Sec. 3, Finally, in Sec. 4 some discussion and conclusions are presented.

\section{LEPTO and Fragmentation Functions}

In the standard picture of SIDIS (see Eq. (10) the quark fragmentation functions by definition depend on the type of hadron, quark flavor and fraction of quark energy carried by hadron, $z$, (there is also a weak dependence on $Q^{2}$ due to perturbative QCD effects), and are independent of $\mathbf{a}$ ) the Bjorken variable $x$ and $\mathbf{b}$ ) the target type. These properties related to universality of fragmentation functions are essential - they indicate that one is dealing with independent quark fragmentation and that there is no influence of the target remnant on hadron production in the current fragmentation region.

These fragmentation function are not well known for different hadron and quark types and the LEPTO event generator is used by HERMES collaboration [1] to calculate purities. 
However, hadronization in this generator is based on the Lund string fragmentation model and one has first to check if the issues a) and $\mathbf{b}$ ) are satisfied in this approach. To this end samples of SIDIS events were generated for HERMES experimental conditions using the settings of LEPTO as in [1] (see also reference [60] of [1]). We use the option LST (8)=0 which corresponds to LO approximation in DIS. The option LST (8)=1 used in reference [60] of [1] corresponds to inclusion in the generation of the first order QCD matrix elements for gluon radiation and photon-gluon fusion. With this option Eq. (11) has to be replaced with the NLO expression to include the gluon distribution and fragmentation functions which are not involved in the LO purity analysis.

In Fig. 1 1 the quark "fragmentation functions" to $\pi^{+}$, extracted from the sample, generated for the HERMES kinematics on a proton target, are presented as a function of $z$. The available range of the Bjorken variable, $x$, was divided into two equally populated inter-

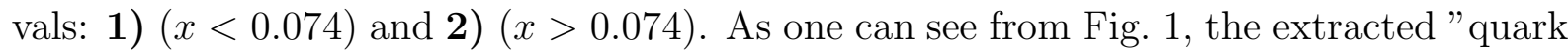
fragmentation functions" happen to be strongly dependent on the Bjorken $x$ variable in striking contradiction with the property a) mentioned above. Note, that this cannot be attributed to the (weak) $Q^{2}$-dependence of fragmentation functions. To demonstrate this the LO fragmentation functions from [4, which includes the QCD evolution, are also presented in the same figure for mean values of $Q^{2}$ corresponding to Bjorken variable intervals 1) $\left(Q^{2}=1.5(\mathrm{GeV} / \mathrm{c})^{2}\right)$ and 2) $\left(Q^{2}=3.4(\mathrm{GeV} / \mathrm{c})^{2}\right)$.

The "quark fragmentation functions" obtained from the samples generated for proton and neutron targets with the cut $x>0.1$ are presented in Fig. 2. We see a dependence on the target type which contradicts property $\mathbf{b}$ ) of fragmentation functions.

Such behavior of "fragmentation functions" extracted from the generated samples is also observed for the production of other types of light meson like $\pi^{-}, K^{+}, K^{-}$etc.

At this point one can conclude that the "quark fragmentation functions" extracted from the samples generated for HERMES kinematical conditions do not correspond to the commonly used notion of fragmentation function. Let us recall that hadronization in the LEPTO event generator is based on string fragmentation and as it is stressed in [3]: "the primary hadrons produced in string fragmentation come from the string as a whole, rather than from an individual parton". In other words the distributions of the produced hadrons "retains the memory" not only of the struck quark type but also of the target remnant and, hence, the entire string configuration. Event generation in LEPTO proceeds in three steps: first, the active parton inside nucleon is chosen according to the parton density function $q\left(x, Q^{2}\right)$, then hard scattering kinematics is generated and finally the string fragmentation machinery of JETSET program [3] is applied to form the final hadrons. Within this approach the SIDIS cross section can be expressed as

$$
\sigma_{N}^{h}\left(x, z, Q^{2}\right) \propto\left(1+(1-y)^{2}\right) \sum_{q} e_{q}^{2} q\left(x, Q^{2}\right) H_{q / N}^{h}\left(x, z, Q^{2}\right)
$$

where the functions $H_{q / N}^{h}\left(x, x_{F}, Q^{2}\right)$ are describing the probability of hadron $h$ production in the hadronization of the system formed by struck quark $q$ and corresponding target remnant. Let us call them LUND hadronization functions), LHF. The target remnant type and, hence, the whole fragmenting system configuration depends both on the nucleon type and on the struck quark type ${ }^{2}$. This means that in contrast with independent

${ }^{2}$ The fragmenting system in LEPTO/JETSET is in general more complicated than a quark-diquark jet. The target remnant state depends on the removed active parton type and the whole fragmenting system 

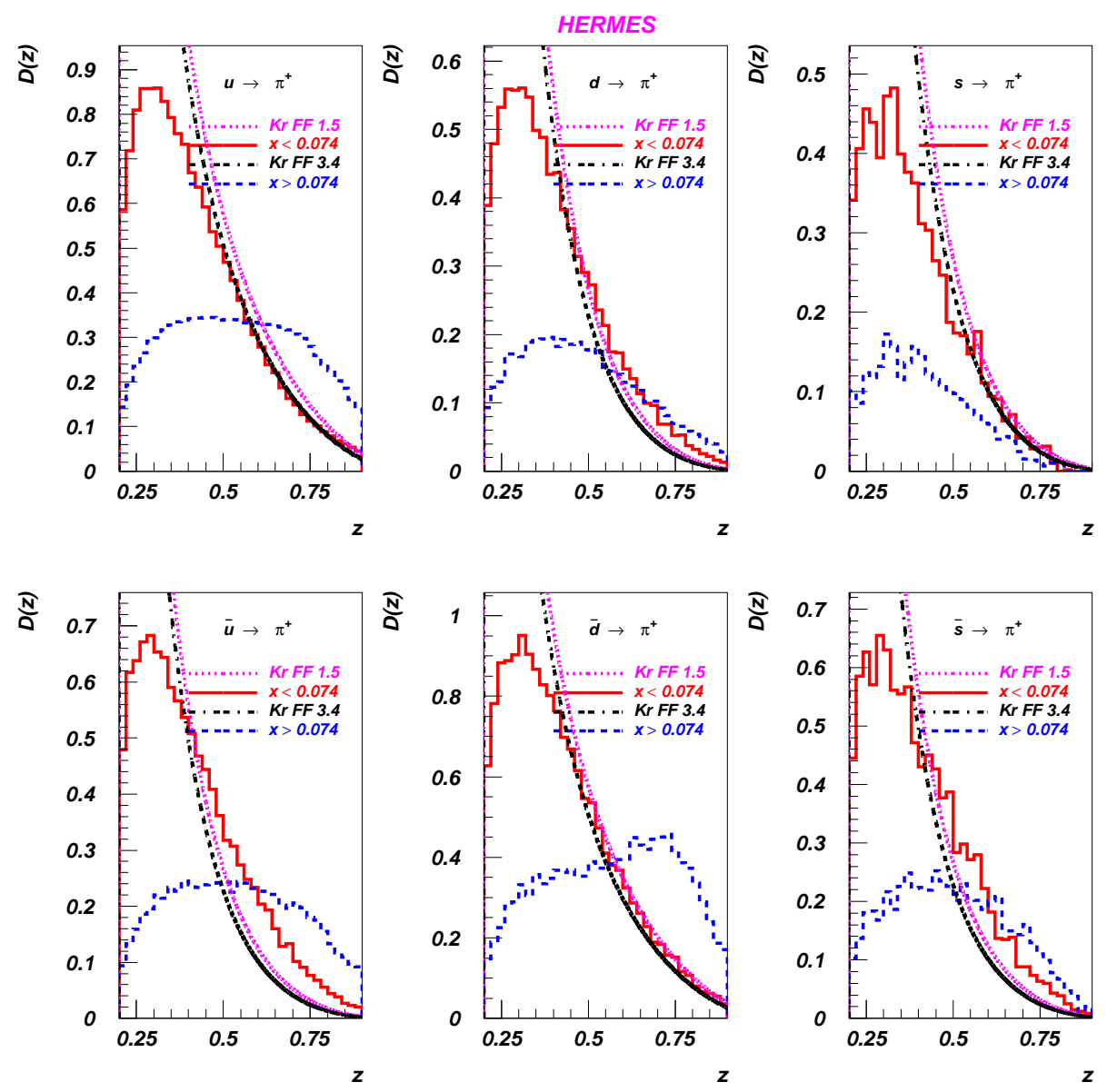

Figure 1: "Quark fragmentation functions" to $\pi^{+}$calculated for HERMES conditions. Solid line - with cut $x<0.074$, dashed line - with cut $x>0.074$. Fragmentation functions from Kretzer: dotted line-for $Q^{2}=1.5(\mathrm{GeV} / \mathrm{c})^{2}$, dot-dashed line-for $Q^{2}=3.4(\mathrm{GeV} / \mathrm{c})^{2}$.

fragmentation functions the LHF's are non universal - they depend on the process type and energy.

Note, that with substitution $z \rightarrow x_{F}$ Eq. (6) is valid not only in the current fragmentation region but in the whole $x_{F}$ interval.

The product $q\left(x, Q^{2}\right) H_{q / N}^{h}\left(x, x_{F}, Q^{2}\right)$ is the conditional probability to find the parton $q$ in the nucleon, $N$, and, after hard interaction, to create a hadron $h$ in the string hadronization. By its physical meaning this object represents nothing else but the fracture functions discussed in [5]. There exist certain arguments based on handbag diagram dominance that this concept may be applied even in the current fragmentation region of SIDIS [6]. The LEPTO/JETSET Monte Carlo program can be considered as a model for these functions. It is clear that from the generated samples one can actually extract only LHF's and as one can see from Fig. 1 and Fig. 2 even in the current fragmentation region one cannot neglect the dependence of this functions on the Bjorken $\mathrm{x}$ variable and on the target type.

Let us now consider the string configuration in more detail in the simplest case when the valence $u$-quark is removed by hard scattering from a proton. Then the target remnant

may contain multi-jet configurations [2] [3]. 

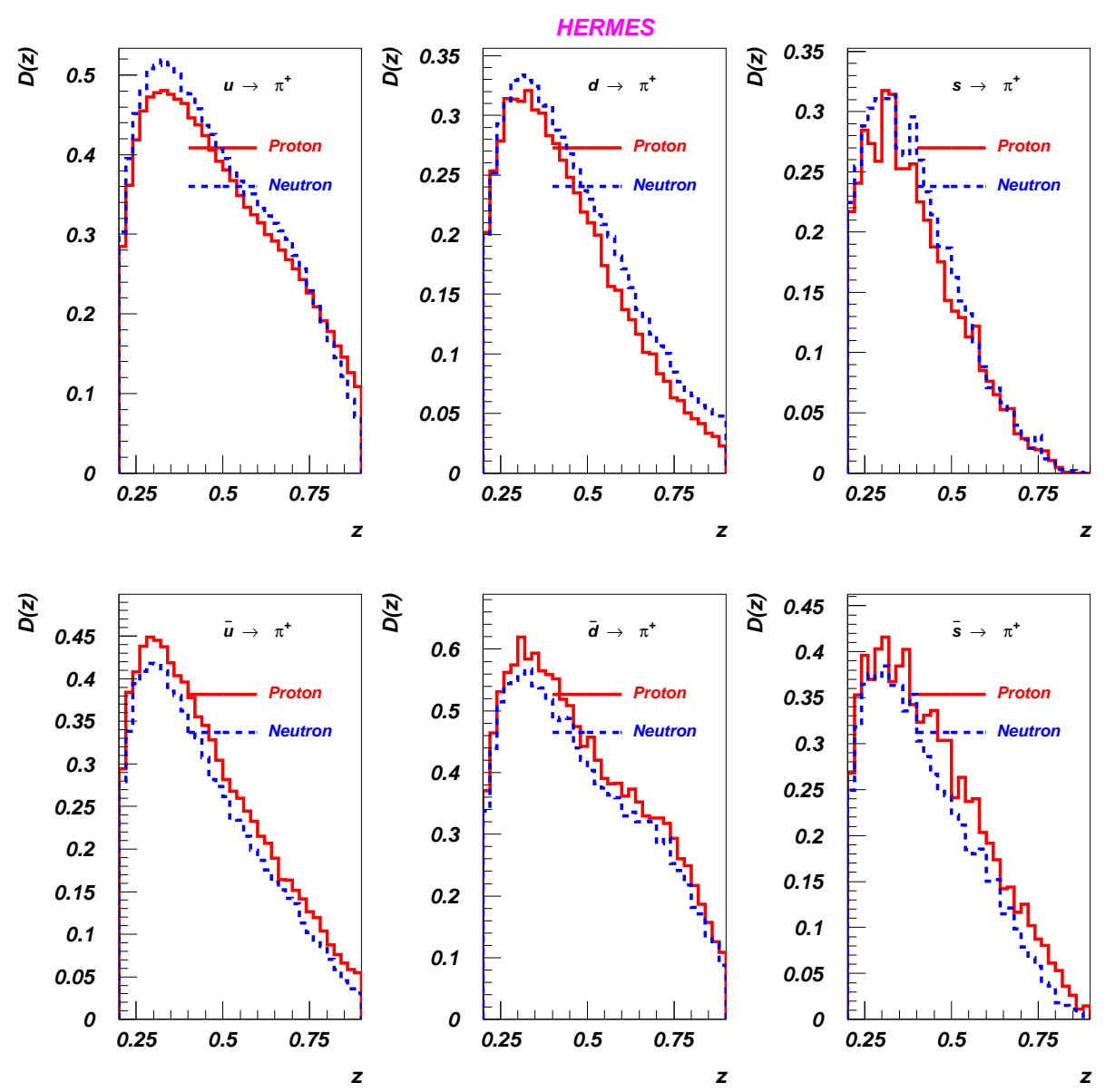

Figure 2: "Quark fragmentation functions" to $\pi^{+}$calculated for HERMES conditions. Solid line - proton target, dashed line - neutron target.

is [2, 3] a scalar, $(u d)_{0}$, diquark or a vector, $(u d)_{1}$, diquark with relative probability $w_{0}=$ 0.75 and $w_{1}=1-w_{0}=0.25$. In Fig. 3 the "fragmentation functions" for $h=\pi^{+}, \pi^{-}, K^{+}$ and $K^{-}$are presented for the cases when the target remnant diquark is chosen to be a $100 \%$ scalar $\left(w_{0}=1\right)$ or a vector one $\left(w_{0}=0\right)$. Let us denote by $\left\{\left(q_{1} q_{2}\right)_{i} \cdots u\right\}-$ the string formed by the struck quark $q$ and the diquark $\left(q_{1} q_{2}\right)_{i}(i=0$ for a scalar diquark and $i=1$ for a vector diquark). We see that LHF's calculated with our generated samples exhibit a dependence on the target remnant spin state $i$ already in unpolarized SIDIS.

\section{Polarized SIDIS and String Fagmentation}

At present there is no the polarized version of the string fragmentation Monte-Carlo program for event generation. It is evident that the description of polarized SIDIS is more complicated than in the unpolarized case. Let us, as an example, again consider the simplest case when the valence $u$-quark with positive $\left(u^{+}\right)$or negative $\left(u^{-}\right)$helicity is removed from nucleon with positive or negative helicity, $N^{+}$or $N^{-}$. Within the $\mathrm{SU}(6)$ 

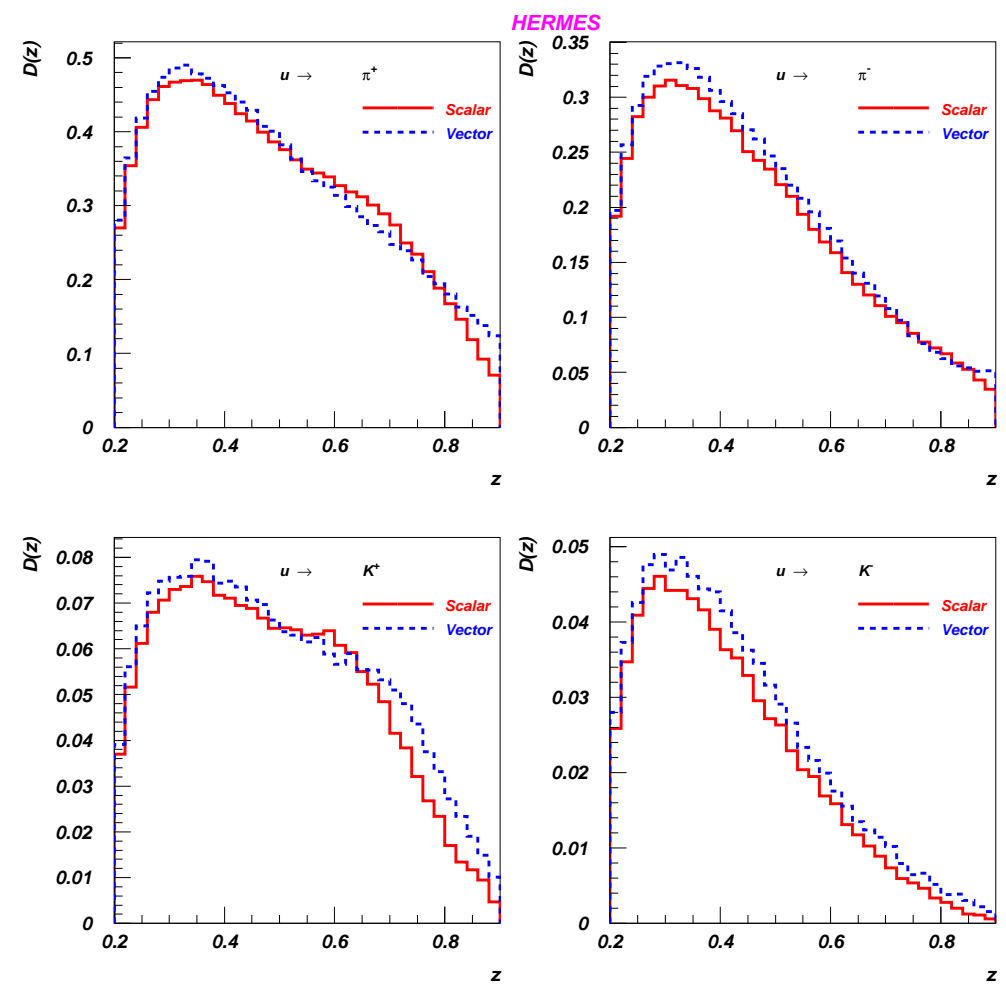

Figure 3: "Quark fragmentation functions" dependence on diquark type calculated for HERMES conditions. Solid line - scalar diquark, dashed line - vector diquark.

quark-diquark model used in [2, 3] the polarized nucleon wave functions are given as

$$
\begin{aligned}
& p^{+}=\frac{1}{\sqrt{18}}\left\{u^{+}\left[3(u d)_{0,0}+(u d)_{1,0}\right]-\sqrt{2} u^{-}(u d)_{1,1}-\sqrt{2} d^{+}(u u)_{1,0}+2 d^{-}(u u)_{1,1}\right\} \\
& n^{+}=\frac{1}{\sqrt{18}}\left\{d^{+}\left[3(u d)_{0,0}+(u d)_{1,0}\right]-\sqrt{2} d^{-}(u d)_{1,1}-\sqrt{2} u^{+}(d d)_{1,0}+2 u^{-}(d d)_{1,1}\right\}
\end{aligned}
$$

where $\left(q_{1} q_{2}\right)_{(i, k)}$ stands for the diquark formed by the $q_{1}$ - and $q_{2}$-quarks with spin $i$ and spin projection $k$.

Using the explicit form of the polarized nucleon wave functions one can calculate the relative probabilities, $w$, of different target remnant states (and hence the states of entire string) depending on the struck quark and the nucleon polarizations. For example, when the $u^{+}$-quark is removed from the $p^{+}$we get the following string configurations with corresponding probabilities $w$

$$
p^{+} \ominus u^{+} \Longrightarrow \begin{cases}\left\{(u d)_{0,0} \cdots \cdot u^{+}\right\}, & w=0.9, \\ \left\{(u d)_{1,0} \cdots \cdot u^{+}\right\}, & w=0.1,\end{cases}
$$

where $\left\{\left(q_{1} q_{2}\right)_{i, k} \cdots \cdots q^{+}\right\}$denotes the string formed by the struck quark $q^{+}$and the diquark $\left(q_{1} q_{2}\right)_{i, j}$. Similarly, when the $u^{+}$-quark is removed from $p^{-}$we get

$$
p^{-} \ominus u^{+} \Longrightarrow\left\{(u d)_{1,-1} \cdots \cdot u^{+}\right\}, \quad w=1 .
$$


For the neutron target we have

$$
n^{+} \ominus u^{+} \Longrightarrow\left\{(d d)_{1,0} \cdots \cdot u^{+}\right\}, \quad w=1
$$

and

$$
n^{-} \ominus u^{+} \Longrightarrow\left\{(d d)_{1,-1} \cdots \cdot u^{+}\right\}, \quad w=1 .
$$

The relations (96,12) demonstrate that the string configuration indeed depends not only on the struck quark type and its polarization but also on the target type and polarization. As we have seen in Sec. 2 the description of hadron production in the current fragmentation region of SIDIS within the LUND fragmentation model does not correspond exactly to the commonly adopted simple picture of independent quark fragmentation but rather to the more complicated approach based on fracture functions. Even in unpolarized SIDIS LHF's depend on target fragment spin states as it is demonstrated in Fig. 3, Then in polarized SIDIS the dependence on the target and struck quark polarizations appears. So, one has to generalize Eq. (6) for the polarized SIDIS case.

Let us start with the SIDIS cross section $\sigma_{N \lambda_{l} \lambda_{N}}^{h}$ for the positive helicity lepton, $\lambda_{l}=+1$ and hadron, $\lambda_{N}=+1$ :

$$
\sigma_{N++}^{h} \propto \sum_{q} e_{q}^{2}\left\{q^{+} H_{q / N++}^{h}+(1-y)^{2} q^{-} H_{q / N-+}^{h}\right\}
$$

where $H_{q / N \lambda_{q} \lambda_{N}}^{h}$ describes the production probability of the hadron $h$ in the quark-target remnant system fragmentation and depends not only on $x$ and $x_{F}$ (or $x$ and $z$ ) but also on the struck quark and nucleon helicities, $\lambda_{q}= \pm 1$ and $\lambda_{N}= \pm 1$. Similarly

$$
\sigma_{N+-}^{h} \propto \sum_{q} e_{q}^{2}\left\{q^{-} H_{q / N+-}^{h}+(1-y)^{2} q^{+} H_{q / N--}^{h}\right\}
$$

The partially polarized beam state, $l^{\lambda_{l}}$, (with helicity $\lambda_{l}$ ) can be described as $l^{\lambda_{l}}=$ $1 / 2\left(1+\lambda_{l}\right) l^{+}+1 / 2\left(1-\lambda_{l}\right) l^{-}$and similarly for the nucleon. Then in the general case of arbitrary polarized beam and target we have

$$
\begin{aligned}
\sigma_{N \lambda_{l} \lambda_{N}}^{h} \propto & \left(1+\lambda_{l}\right)\left(1+\lambda_{N}\right) \sigma_{N++}^{h}+\left(1+\lambda_{l}\right)\left(1-\lambda_{N}\right) \sigma_{N+-}^{h}+ \\
& \left(1-\lambda_{l}\right)\left(1+\lambda_{N}\right) \sigma_{N-+}^{h}+\left(1-\lambda_{l}\right)\left(1-\lambda_{N}\right) \sigma_{N--}^{h} .
\end{aligned}
$$

Now, using the relations $H_{q / N++}^{h}=H_{q / N--}^{h}$ and $H_{q / N+-}^{h}=H_{q / N-+}^{h}$ which follow from parity invariance and introducing

$$
\begin{aligned}
H_{q / N}^{h} & =H_{q / N++}^{h}+H_{q / N+-}^{h} \\
\Delta H_{q / N}^{h} & =H_{q / N++}^{h}-H_{q / N+-}^{h}
\end{aligned}
$$

after simple algebra one get:

$$
\begin{gathered}
\sigma_{N \lambda_{l} \lambda_{N}}^{h} \propto\left[1+(1-y)^{2}\right] \sum_{q} e_{q}^{2}\left\{q H_{q / N}^{h}+\Delta q \Delta H_{q / N}^{h}\right\}+ \\
\lambda_{l} \lambda_{N}\left[1-(1-y)^{2}\right] \sum_{q} e_{q}^{2}\left\{\Delta q H_{q / N}^{h}+q \Delta H_{q / N}^{h}\right\}
\end{gathered}
$$


where now $\lambda_{l}$ and $\lambda_{N}$ are the (arbitrary) beam and target helicities.

Eq. (17) is very similar to the equation proposed in [7]. The difference is that functions

$H_{q / N}^{h}$ and $\Delta H_{q / N}^{h}$ are not independent quark fragmentation functions like in [7] but LHF'sthey describe the probability of hadron production in the hadronization of the whole struck quark - target remnant system. Even for hadrons produced in the current fragmentation region these functions can depend not only on the fraction of the quark energy carried by produced hadron but also on the whole hadronic CMS energy and the target and the struck quark polarizations.

When integrated over the whole available phase space of the selected hadron, Eq. (17) transforms to the standard expression for the polarized DIS, provided that the following sum rules hold valid:

$$
\begin{aligned}
& \int_{-1}^{1} d x_{F} H_{q / N}^{h}=\left\langle n^{h}\right\rangle \\
& \int_{-1}^{1} d x_{F} \Delta H_{q / N}^{h}=0
\end{aligned}
$$

where $\left\langle n^{h}\right\rangle$ represents the mean multiplicity of the hadron $h$.

\section{Discussion and Conclusions}

The standard expression for the SIDIS description in the current fragmentation region is obtained if one assume that

$$
H_{q / N}^{h}\left(x, z, Q^{2}\right) \rightarrow D_{q}^{h}\left(z, Q^{2}\right)
$$

and

$$
\Delta H_{q / N}^{h}\left(x, z, Q^{2}\right) \rightarrow 0 .
$$

As we have demonstrated in Sec. 2 relation (19) is not correct for the HERMES experimental conditions in the LUND fragmentation model. On the other hand we have seen that hadronization in this model depends on the target remnant spin quantum numbers even in the simplest case of a removed valence quark, see Fig. 3. In the case of polarized SIDIS the relative probabilities of different target remnant states depend on the target and struck quark polarizations, see Eqs. (9,10). So, there is no any reason to believe that relation (201) will hold for the polarized SIDIS at moderate energies. Thus, a new nonperturbative input - the polarized $L H F$ 's, $\Delta H_{q / N}^{h}\left(x, z, Q^{2}\right)$ is needed. In this case Eq. (3) underlying the purity method is based is not exact and must to be replaced by

$$
A_{1}^{h}\left(x, Q^{2}, z\right)=\frac{\sum_{q} e_{q}^{2}\left[\Delta q\left(x, Q^{2}\right) H_{q / N}^{h}\left(x, z, Q^{2}\right)+q\left(x, Q^{2}\right) \Delta H_{q / N}^{h}\left(x, z, Q^{2}\right)\right]}{\sum_{q} e_{q}^{2}\left[q\left(x, Q^{2}\right) H_{q / N}^{h}\left(x, z, Q^{2}\right)+\Delta q\left(x, Q^{2}\right) \Delta H_{q / N}^{h}\left(x, z, Q^{2}\right)\right]},
$$

with extra contributions in the numerator and denominator as compared to Eq. (3).

In [9] a model for the extra contribution in the numerator of Eq. (21) has been considered and it was demonstrated that our ignorance of polarized LHF's may lead to incorrect results for polarized sea quark distributions.

It is interesting to check the properties of the "quark fragmentation functions" calculated from samples generated with LEPTO event generator at higher energies. In Fig. 4 

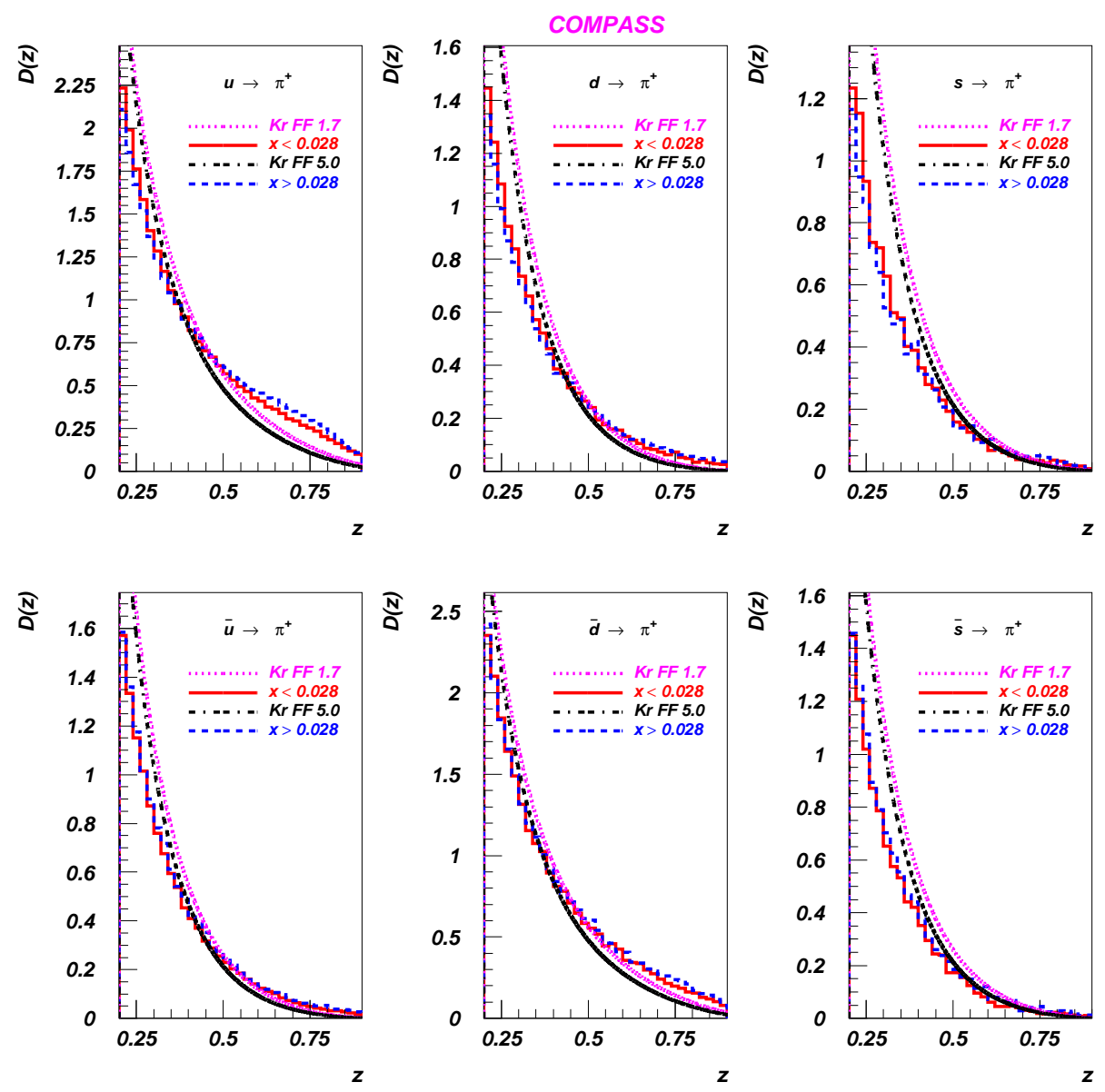

Figure 4: "Quark fragmentation functions" to $\pi^{+}$calculated for COMPASS conditions. Solid line - with the $x<0.028$, dashed line - with cut $x>0.028$. Fragmentation functions from Kretzer: dotted line-for $Q^{2}=1.7(\mathrm{GeV} / \mathrm{c})^{2}$, dot-dashed line-for $Q^{2}=5.0(\mathrm{GeV} / \mathrm{c})^{2}$.

the same distributions as in Fig. 1] are presented for COMPASS [8] kinematics. One can see that the dependence on Bjorken variable of the "quark fragmentation functions" extracted from generated samples is less pronounced that in case of HERMES experimental conditions. The same observation is also valid for the dependence upon the target type and the target remnant spin state dependencies. This means that that polarized LHF's may be negligible in the current fragmentation region at high energies.

The string configurations considered in (9, 12) correspond to the simplest case of removing the valence quark from nucleon. In the case, when the virtual photon interacts with the sea quark or higher order hard scattering processes are considered, the target remnant and the final parton configuration are more complicated 22. For example, in photon-gluon fusion the target remnant is split into a quark and a diquark that form two respective separate strings with the antiquark and quark produced in the fusion process. One can generalize Eq. (17) to include the corrections from higher order QCD hard processes. The generalized expression for the polarized cross section of single and two hadron production will, in addition, contain new unknown LHF's, $\Delta H_{g / N}^{h}\left(x, z, p_{T}^{h}, Q^{2}\right)$ and $\Delta H_{g / N}^{h_{1}, h_{2}}\left(x, z_{1}, z_{2}, p_{T}^{h_{1}}, p_{T}^{h_{2}}, Q^{2}\right)$, with the corresponding distribution functions, $(\Delta) g\left(x, Q^{2}\right)$. This means that the validity of the Monte Carlo based approach [10] to extract the po- 
larized gluon distribution may also be questionable at moderate energies.

It has been recently noted [6] that the appearance of separate distribution and fragmentation functions cannot be proven in general, but is rather assumed and justified a posteriori, while the natural framework to describe SIDIS involves fracture functions. These functions can be also generalized to describe the T-odd single spin asymmetries [6]. As it was demonstrated above the fracture functions within the LUND fragmentation framework are represented by sums of products of distribution functions and LHF's. Recent developments in the theory of SIDIS for single spin asymmetries and diffractive phenomena also show that one cannot neglect the interaction of (colored) removed partons and target remnants (see, for example, [1] and references therein, and [6]). This again indicates that the description of SIDIS based on the naïve parton model with the independent fragmentation is only an approximation, to be justified at moderate energies.

\section{Acknowledgements}

The author express his gratitude to M. Anselmino, A. Efremov and O. Teryaev for discussions, G. Pontecorvo for careful reading of the manuscript and also to NUCLEOFIT group members of the General Physics Department "A. Avogadro" of the Turin University for interest in this work.

\section{References}

[1] A. Airapetian et al., [HERMES Collaboration], arXiv:hep-ex/0407032.

[2] G. Ingelman, A. Edin and J. Rathsman, Comp. Phys. Commun. 101108 (1997).

[3] T. Sjöstrand, Comp. Phys. Commun. 39347 (1986), 43367 (1987);

T. Sjöstrand, PYTHIA 5.7 and JETSET 7.4: Physics and Manual, arXiv:hep-ph/9508391

T. Sjöstrand, PYTHIA 6.2: Physics and Manual, arXiv:hep-ph/0108264

[4] S. Kretzer, Phys. Rev. D 62 (2000) 054001.

[5] L. Trentadue and G. Veneziano, Phys. Lett. B323 201 (1994).

[6] O. V. Teryaev, Acta Phys. Polon. B 33, 3749 (2002), arXiv:hep-ph/0211027;

Proceedings of X Advanced Research Workshop on High Energy Spin Physics, Dubna, September 16-20, 2003, p. 200.

[7] M. Gluck and E. Reya, arXiv:hep-ph/0203063.

[8] The COMPASS Proposal, CERN/SPSLC 96-14, SPSC/P297, March 1996.

[9] A. Kotzinian, Phys. Lett. B 552 (2003) 172, arXiv:hep-ph/0211162.

[10] A. Bravar, D. von Harrach and A. Kotzinian, Phys. Lett. B 421 (1998) 349, arXiv:hep-ph/9710266

[11] S. J. Brodsky, R. Enberg, P. Hoyer and G. Ingelman, arXiv:hep-ph/0409119. 